\title{
Germanica
}

\section{Au pays des contes, récit de Knut Hamsun : un voyage intérieur aux sources de son inspiration}

Im Märchenland, von Knut Hamsun, Bericht einer inneren Reise zu den Quellen seiner Inspiration

\section{Marc Auchet}

\section{OpenEdition}

\section{Journals}

Édition électronique

URL : http://journals.openedition.org/germanica/2184

DOI : 10.4000/germanica.2184

ISSN : 2107-0784

\section{Éditeur}

Université de Lille

\section{Édition imprimée}

Date de publication : 31 décembre 2001

Pagination : 11-35

ISBN : 9782913857063

ISSN : 0984-2632

\section{Référence électronique}

Marc Auchet, «Au pays des contes, récit de Knut Hamsun : un voyage intérieur aux sources de son inspiration », Germanica [En ligne], 29 | 2001, mis en ligne le 20 septembre 2013, consulté le 06 octobre 2020. URL : http://journals.openedition.org/germanica/2184 ; DOI : https://doi.org/10.4000/ germanica. 2184

Ce document a été généré automatiquement le 6 octobre 2020.

(C) Tous droits réservés 


\title{
Au pays des contes, récit de Knut Hamsun : un voyage intérieur aux sources de son inspiration
}

\author{
Im Märchenland, von Knut Hamsun, Bericht einer inneren Reise zu den \\ Quellen seiner Inspiration
}

Marc Auchet

1 Le but essentiel du présent article est de montrer que l'ouvrage que Knut Hamsun a publié en 1903 sous le titre Au pays des contes est avant tout le récit d'un voyage intérieur, presque initiatique, très révélateur quant aux sources qui ont inspiré l'ensemble de son œuvre. Écrit à la première personne, ce récit se présente de prime abord comme le compte rendu du voyage qu'il a fait en 1899, de Saint-Pétersbourg à Batoum, en Géorgie, en compagnie de sa première femme, Bergljot Goepfert. Le soustitre signale que c'est leur passage dans le Caucase qui en constitue le principal centre d'intérêt. Avant de lui donner son titre définitif, Hamsun a d'ailleurs longtemps désigné ce texte sous le nom de Livre sur le Caucase (Kaukasusbogen).

Dans Processen mod Hamsun (Le procès contre Hamsun, 1979), Thorkild Hansen estime que ce voyage en Orient a été « déterminant» (bestemmende) pour le romancier, et qu'il a même été « le tournant de sa vie » (vendepunktet $\mathrm{i}$ hans liv). C'est surtout la découverte d'un mode de vie contemplatif, celui des pauvres paysans de ces lointaines régions musulmanes, par opposition au culte de l'argent et de la production industrielle propre à l'Amérique, qui aurait produit un impact particulier sur l'esprit du voyageur. «C'est ici que se trouve la formule qui rend compte de l'ensemble de son œuvre, le conflit fondamental exprimé et défini une fois pour toutes [...], le drame de notre époque et de notre globe : une vie pauvre mais porteuse de sens par opposition à une vie riche qui en est dépourvue ${ }^{1}$. » Atle Kittang estime de son côté que ce point de vue n'est « pas du tout dénoué de fondement ${ }^{2}$ ». Les pages qui suivent devraient le confirmer. Si les commentateurs ont consacré peu d'attention à ce texte jusqu'à présent ${ }^{3}$, cela tient sans doute au fait que son message est crypté. Nous espérons pouvoir montrer ici qu'il a une 
forte charge symbolique et qu'une analyse des images qu'il renferme permet de lui arracher une partie de son « secret ».

\section{Le narrateur et sa « muse »}

On sait que le mariage de Hamsun avec Bergljot Goepfert a été tout sauf harmonieux. Le divorce a été prononcé en 1906, après huit années d'une vie conjugale difficile, pendant laquelle les deux époux ont souvent été séparés. La période la plus longue pendant laquelle ils ont vécu ensemble a duré de la fin de 1898 à l'automne 1899. Elle correspond au séjour qu'ils ont fait en Finlande, et au voyage de quelques semaines qui les a menés en Russie, dans le Caucase et en Turquie. Si la jeune femme a été incapable de créer un foyer agréable pour Hamsun, elle n'a pas non plus été pour lui une source d'inspiration, à une notable exception près: le roman Victoria, publié en $1898^{4}$. C'est peut-être le "froid» qui régnait dans le couple que traduit une remarque un peu agacée du narrateur de Au pays des contes à l'égard de sa compagne de voyage, dont il dévoile incidemment la véritable identité dans les premières pages de son livre : «Et nous voilà bel et bien sortis de la gare pétersbourgeoise. Ma femme n'oublia qu'une chose : son manteau ${ }^{5}$. (p. 13) Du début à la fin du récit, il manifeste une absence totale d'intérêt pour elle, la rabroue même parfois sans beaucoup de ménagement ${ }^{6}$. L'impression générale qui se dégage du texte est que les deux époux vivent chacun dans leur univers. L'auteur ne donne d'ailleurs pratiquement jamais la parole à sa femme, sauf au chapitre XII, où il lui fait dire qu'elle doute de sa « capacité d'écrire un excellent journal de voyage ». Il est bon de noter que l'écrivain (en réalité : le narrateur) réagit alors violemment. «Et ce fut alors que la coupe déborda.» Il quitte la chambre, «le cœur mauvais » (p. 168), et part à la découverte de la ville où se trouve leur hôtel, puis se lance dans un long exposé sur la littérature russe, qui est l'un des passages-clés de tout l'ouvrage.

4 Au début de ce même chapitre, le lecteur apprend que pendant tout son voyage, le narrateur n'a eu qu'une lecture : un vieux numéro de journal qu'il n'a cessé de lire et relire ${ }^{7}$. Fait insolite, au moment précis où se déroule la scène qui introduit ce passage, le touriste-écrivain découvre sa femme en train de lire un livre sous la lampe. «J'étais trop las et trop mal en point, de fièvre, pour m'enquérir de ce que signifiait cette extravagance. [...] On ne devrait jamais avoir de compagne de voyage; votre compagne ne songera qu'à ses aises, et les meilleurs morceaux, c'est elle qui vous les raflera!» Quelques instants plus tard, il lui "lance quelques mots au hasard», et l'absence de communication est telle qu'il a littéralement le sentiment de parler à un mur. C'est alors que sa femme lui pose quelques questions précises, qui lui font comprendre que c'est en fait son journal de voyage qui lui a servi de lecture cette nuit-là. (p. 167-168) Ce bref passage permet d'identifier la problématique générale dont le livre donne une transcription littéraire: il s'agit pour l'auteur d'un retour aux sources de la création artistique. Au lieu d'être sa muse, cette femme qui voyage avec lui essaye de le faire douter de son talent et le paralyse. Elle symbolise une situation de stagnation quant à l'écriture. 


\section{La problématique de l'écriture - les modèles russes}

5 Le sous-titre que Hamsun a choisi pour Au pays de contes est éloquent: « choses vécues et choses rêvées au Caucase ». Il signale par là qu'il s'agit de tout autre chose qu'une relation de voyage traditionnelle, et la « fièvre caucasienne » qui poursuit le narrateur pratiquement d'un bout à l'autre du récit est un artifice facile qui lui permet de s'affranchir du carcan du réalisme et de se livrer à toutes les fantaisies. Il est essentiel de comprendre que les faits rapportés se situent souvent dans un monde onirique ou relevant d'une psychologie proche du pathologique, dans le droit fil du programme énoncé dans l'essai «De la vie inconsciente de l'âme", et des romans comme Faim, Mystères ou Pan. L'auteur prend visiblement plaisir à se moquer de la tendance réaliste en littérature, en particulier lorsqu'il fait allusion au caractère "scientifique » de ses observations. Dès le départ, il signale qu'il fait ce voyage grâce à une «bourse d'État " (statsstipendium), mais il émaille son texte de remarques plaisantes sur les travaux savants: il traite les connaissances linguistiques avec beaucoup de condescendance, alors qu'il est incapable de parler correctement une autre langue que le norvégien, se pose en anthropologue averti, prêt à "édifier toute une théorie nouvelle sur la migration des peuples" (p.84) ou à faire une communication importante devant ses pairs dans le cadre d'une hypothétique "Société de Géographie », bien qu'il ne dispose que de quelques observations fortuites rassemblées en toute hâte, etc. Le passage le plus caractéristique à cet égard est le récit qu'il fait de sa visite de l'usine Nobel, à Bakou. Il prétend avoir été contraint de prendre des notes en tenant son carnet dans son dos et d'avoir ensuite mélangé toutes ces informations avec une grande désinvolture au moment de la relecture (p. 200-201). On peut difficilement imaginer un ton plus moqueur pour jeter le discrédit sur le réalisme en littérature.

6 Il oppose d'ailleurs clairement les lectures stériles des peuples de l'Occident, "les romans et le journal », à la culture des peuples anciens, qui ne lisent pas, mais « rôdent dans la nuit en grattant des chansons » (p. 62). La littérature qu'il appelle de ses vœux est représentée par plusieurs des grands auteurs russes, dont les "personnages sont mus par cette spontanéité, cette faculté du déraillement, cette aptitude à l'absurde dont le peuple russe dispose seul»(p.171). C'est la raison pour laquelle il voue une admiration sans bornes à Dostoïevski, qui «s'en alla en fanatique, en fou, en esprit génial. Il était aussi surexcité et déraciné que le sont ses personnages [...] son sens psychologique est accablant, visionnaire. Pour l'apprécier, on manque de la mesure selon laquelle l'évaluer, il est unique. Ses contemporains voulurent le calibrer, mais n'y réussirent pas; il est trop grand, il déborde [...] À la première entrevue qu'il [un critique d'art] eut avec Dostoïevski, il appréciait chaleureusement le jeune écrivain ; mais celui-ci déplut tout de suite au grand critique, en se considérant comme un génie. Tout simplement» (p. 173). Hamsun reprend l'argument principal de la conférence qu'il avait faite à Helsinki, lors de son séjour en Finlande : on surestime généralement les poètes et la littérature en les considérant comme des penseurs, comme "les directeurs spirituels des peuples» (p. 178). Il en profite aussi pour donner au passage un coup de griffe à la période des années soixante-dix, c'est-à-dire au mouvement de la Percée Moderne, qui, dans le sillage de Georg Brandes ${ }^{8}$, a - selon lui - détourné les poètes de leur mission. Ils avaient été jusque-là " des chanteurs, des fournisseurs de sensations, des conteurs ", mais on a fait d'eux "des travailleurs, des éducateurs, des réformateurs [...] Les poètes devinrent des personnages ayant une opinion sur toute 
chose » (p. 177). Le voyage en Russie l'a mis en contact direct avec un peuple inspiré, avec le " pays des contes".

7 Comme nous l'avons déjà signalé, c'est surtout le Caucase qui fascine le narrateur. «Le Caucase, le Caucase! Ce n'est pas pour rien que les plus absolus géants poétiques que connaisse le monde, les grands Russes, sont allés chez toi puiser à tes sources... » (p.137). La valeur mythique de cette région est signalée lors du passage du couple à Tiflis, au cours duquel il consacre beaucoup de temps à la visite du «quartier asiatique ». Le narrateur s'attarde en particulier sur le compte d'un écrivain public, qu'il considère "avec respect », et dont il apprécie la "charmante dignité ». "Il a autour de lui des livres aux lettres bizarres; ce n'est pas étonnant qu'il soit devenu si gris et si digne, pensons-nous, puisqu'il a appris à écrire de telles lettres et sait les déchiffrer. " Il décrit ensuite comment des jeunes gens qu'il prend pour des érudits s'inclinent et saluent respectueusement cet écrivain public. "L'art d'écrire est un art sacré, le papier même sur lequel on écrit est sacré. Le glorieux cheik Abd-ul-KaderGilani ne passait jamais devant une papeterie sans s'être préalablement purifié par des ablutions [...] En somme, l'art d'écrire et de lire est, semble-t-il, en honneur dans l'Islam. » Le narrateur mentionne avec quelque prudence l'avis d'un spécialiste selon lequel les universités du monde musulman seraient en décadence, mais il prend soin de souligner que « dans l'Asie centrale il existe encore des contrées où il y a des académies d'une si grande renommée qu'elles attirent des élèves de l'Arabie, de l'Inde, du Cachemire, de la Chine, même des bords de la Volga. Et chez des particuliers, on peut rencontrer parfois une érudition très grande, cela va sans dire.» Ce passage qui attribue un caractère sacré à l'écriture, même - et peut-être surtout - si elle est séparée de l'érudition, a certainement une forte valeur symbolique de la part d'un auteur chez qui l'acte d'écriture semble avoir eu un caractère compulsif et obsessionnel ${ }^{9}$. Il voue à la littérature russe une admiration qu'il étend à l'ensemble du peuple. « Des Slaves! me dis-je en les regardant, le peuple de l'avenir, les vainqueurs du monde après les Germains! D'un tel peuple, une littérature comme celle de la Russie peut jaillir, puissante, illimitée, tumultueuse, aux huit sources impétueuses de leurs huit poètes géants» (p. 26). Il est toutefois évident que son admiration pour la Russie ne se limite pas à des questions purement littéraires.

\section{La Russie : un décor digne des contes de fées}

8 Dès les premiers pages de son récit, lorsqu'il décrit Saint-Pétersbourg, au moment où il va entrer dans la "grande Russie », le narrateur mentionne son intérêt pour le culte des icônes. «Là je vois pour la première fois des lampes qui brûlent devant les saintes images. » Il oppose aussitôt ces « lampes éternelles [qui] brûlent, nuit et jour, devant les icônes [...], pareilles à de petits autels» au «tintamarre des wagons et des locomotives", qu'il assimile à "l'Amérique qui beugle» (p.12-13). L'opposition fondamentale, déjà signalée, entre une civilisation contemplative et un monde où la technique règne en maître, est ainsi clairement posée dès le début. Le thème de l'icône revient d'ailleurs un peu plus loin, au moment où le narrateur mange seul dans un restaurant et décide tout à coup de se lever et d'aller s'incliner devant une image sainte, comme les autres clients. «La joie de me trouver dans ce grand pays, dont j'ai tant lu, me remplit tout entier et cette joie se traduit par une surexcitation intérieure 
qu'en ce moment je ne me soucie pas de maîtriser» (p. 28). La Russie, c'est donc le monde des icônes, des images et des symboles, le monde du rêve et de l'imaginaire.

Moscou est comparée à l'univers des "contes de fées " où l'on est "ébloui par de fabuleuses richesses» (p.20), mais le décor grandiose du Caucase exerce une fascination plus forte encore sur le narrateur. Il est comme sous le coup d'un enchantement. «Tout cela surgit du sol noir, au beau milieu de la steppe. Par le soleil vibrant, on dirait que c'est suspendu au-dessus de la terre, que le château plane sur l'horizon et au-delà. Une fantasmagorie... » (p. 50). « Au loin se profilent les montagnes neigeuses, qui se fondent presque avec les nuages blancs du ciel. C'est comme un conte de fées » (p. 56). Arrivé dans les montagnes, il a même le sentiment d'être en contact avec un autre monde. "Tout à coup la route tourne brusquement, un gouffre immense s'ouvre à droite, et tout près de nous apparaît le glacier de Kazbek, dont la blancheur étincelle au soleil. Il nous touche presque. Un sentiment étrange nous secoue; cette blanche montagne nous semble ainsi dressée de par la volonté magique des monts ; elle nous paraît être d'une autre planète, qui est là et qui nous regarde [...] En ce moment, une impression vertigineuse me saisit tout entier, je me sens soulevé de la route, jeté hors de mes gonds; c'est comme si je me trouvais face à face avec un dieu » (p. 79). Le point culminant est atteint ${ }^{10}$ lors d'une promenade à cheval qu'il fait seul dans les montagnes, à quelque deux mille mètres d'altitude, en pleine nuit. «Jamais je n'ai vu les étoiles briller d'un éclat aussi vif qu'ici, parmi les monts du Caucase. La lune n'est qu'un croissant, mais elle brille comme si elle était pleine. C'est une chose neuve pour moi que cet éclat intense d'un ciel nocturne sans soleil [...] Quel monde profond et sorcier ['trollsk', ensorcelé serait sans doute préférable] que celui-ci! Cet ancien lieu d'exil est bien le plus merveilleux des pays. Je m'abandonne de plus en plus et ne pense guère au sommeil. Les montagnes me paraissent fantastiques; on les dirait venues d'autre part pour s'arrêter juste devant moi » (p. 98). Ce Caucase qui regorge de fruits succulents, c'est plus qu'un pays de Cocagne, c'est presque une Terre promise, un Paradis ${ }^{11}$. «La végétation est si abondante que je n'ai rien vu de semblable. Les forêts semblent impénétrables, et lorsque nous nous arrêtons aux gares et que nous pouvons regarder d'un peu plus près, nous apercevons un fouillis de lianes à l'aspect de forêt vierge. Ici poussent le châtaignier, le noyer, le chêne, et, comme taillis, des noisetiers. Dans les espaces défrichés, on cultive le maïs, la vigne et quantité de fruits; tout cela pousse, d'une sève exubérante, mûrit sur tige; l'air est embaumé du parfum des pommes. Nous plongeons nos regards dans ce divin pays sans pareil ; comme c'est riche et beau! Et avoir pu le contempler! La lune est déjà levée avant que le soleil se couche, les étoiles jaillissent par traînées ici aussi, et le train s'enfonce, nageant dans une mer argentée dans l'outre-terre »(p. 228-229).

\section{L'opposition Orient-Occident}

Le décor n'est évidemment pas tout. Le peuple qui y vit est porteur de valeurs qui, aux yeux du narrateur, sont infiniment supérieures à celles de l'Occident. "Ce monde ne ressemble à nul autre que je connaisse, et je me reprends à souhaiter de vivre ici ma vie. [...] On demeure bouleversé devant cette splendeur et si l'on vivait ici, on pourrait la contempler chaque jour et d'émerveillement se frapper la poitrine. Ce peuple a enduré des combats qui menacèrent de le dévaster, mais il a tout surmonté, et il est fort et sain, il fleurit, il est actuellement un peuple de dix millions d'hommes. Le Caucasien 
ne connait certes pas les fluctuations de la Bourse de New-York, son existence n'est pas une course de vitesse, il a le temps de vivre; et son manger peut se cueillir sur les arbres, ou bien il peut abattre son mouton pour se nourrir. Mais l'Européen et le Yankee sont pourtant de plus grands hommes? Dieu sait. Dieu et nul autre ne le sait, tant c'est douteux. [...] Les valeurs n'ont pas une signification immuable : un nimbe de théâtre, là-bas, correspond à une ceinture brillante, par ici ; le temps s'en empare, le temps les change pour d'autres valeurs. Le Caucase, le Caucase !» (p. 136-137). Comme Hamsun l'écrira à nouveau dans son essai "Festina lente ", en 1928, l'Orient, c'est le calme, l'Occident, c'est le brouhaha qui caractérise la civilisation américaine, obsédée par le gain. «Le progrès, c'est le repos nécessaire du corps et le calme nécessaire de l'âme. Le progrès, c'est le bien-être de l'être humain ${ }^{12}$."

11 Pour le narrateur, l'influence de l'Islam est un facteur déterminant qui explique le stoïcisme et le calme des populations concernées. « Plus on approche de l'Orient, moins les hommes parlent. Les races anciennes ont dépassé la phase du bavardage et du rire bruyant; les gens se taisent et sourient. Peut-être cela vaut-il mieux. Le Coran a développé une attitude devant la vie qui se prête mal aux meetings et aux discussions. Le sens en est un : le bonheur, c'est d'endurer la vie jusqu'à son terme; après, ce sera mieux. Le fatalisme. » En entendant un homme gratter les cordes de sa balalaïka, il estime que c'est une "musique de la vie fossile », un retour à la vie végétative. "C'est une joie douce qu'il offre à tous, et à lui-même, puisqu'il continue. Quel étrange peuple en ce pays étrange! Ils ont le temps de faire de la musique, le don de savoir se taire! Dieu soit loué qu'il existe de tels pays sur la terre! Et ils ne pourraient avoir de meilleurs voisins que le Slave, car le Slave a lui-même une corde dans la poitrine.» (p. 61)

12 Le charme du quartier asiatique de Tiflis, réside dans le fait que « tout y était paisible, [...] personne ne se hâtait, le calme de l'Orient régnait sur les hommes» (p. 184). Les commerçants y vaquent à leurs occupations "lentement et majestueusement " (p. 185-186). «Un Oriental n'est pas si empressé - à moins qu'il n'ait été corrompu par l'esprit occidental »(p.186), et le narrateur n'hésite pas à parler à l'occasion de "démoralisation européenne » (p.225). "Le fatalisme, vieille philosophie éprouvée, système simple et absolu, a son foyer en Orient [...] Sa doctrine est simple et résistante, elle est de fer.» (p. 190) L'insistance que met le touriste-écrivain à vanter la supériorité de la mentalité orientale et de la religion musulmane ${ }^{13}$ montre que sa conception de l'Orient se rattache bien à quelque "géographie spirituelle » qui se déploie avant tout dans le monde de l'âme, dans le mundus imaginalis ${ }^{14}$. C'est ce qui nous faisait écrire au début de cet article que le voyage au Caucase est pour une bonne part un voyage initiatique.

\section{Le symbolisme du feu}

13 Comme nous l'avons déjà signalé, le feu joue dès le début du récit un rôle symbolique important, sous la forme des "lampes éternelles » qui, " pareilles à de petits autels ", «brillent d'un éclat adouci » devant les icônes. Le lecteur apprend que « les Russes font le signe de la croix en passant devant les images » et « ne partent jamais en voyage sans accomplir cette cérémonie» (p.12-13). Tout se passe comme si le narrateur voulait absolument faire comprendre à son lecteur qu'il entre dans un monde régi par d'autres lois que celles du monde moderne. 
14 Après cette première occurrence, qui signale aussi d'emblée l'importance du symbolisme igné pour l'ensemble du récit ${ }^{15}$, il faut attendre le chapitre six pour que celui-ci réapparaisse de façon explicite. C'est le moment où le narrateur s'exprime sur le compte des Ossètes, « ce peuple dont personne n'a su pénétrer l'origine ni le nom; il se nomme lui-même Iron ». Le texte renferme alors quelques remarques volontairement naïves sur les méthodes de travail qu'il entend utiliser, et qui discréditent ironiquement ce genre de recherches. Le passage le plus important est en rapport direct avec la problématique de tout le livre: «Ici se trouve le berceau de l'humanité, ici Prométhée fut lié au rocher, ici brûle le feu éternel, dans les environs de Bakou... » (p. 83). Le lecteur apprend ainsi inopinément que le «feu» qui préoccupe tant le narrateur est en réalité celui que Prométhée a dérobé aux dieux, qui, on le sait, symbolise souvent l'inspiration poétique.

15 Le chapitre XVI est entièrement consacré au thème du feu ${ }^{16}$. Il a une importance centrale pour notre analyse. Le narrateur y décrit une visite aux sources de pétrole de Balakani. Il ne cache pas que la "ville de tours de forage " qu'il découvre à cette occasion est « la ville la plus incroyable et la plus déplaisante que l'on puisse imaginer ; elle est composée entièrement de tours noires, graisseuses, en charpentes sommaires. À l'intérieur, des machines font rage, jour et nuit...» (p. 209). Mais il précise dans un passage particulièrement révélateur que «cet endroit n'a pas toujours connu le vacarme des machines. C'est l'Amérique qui est venu le profaner, c'est le foyer du "feu éternel" de l'antiquité.» Regrettant amèrement que "nulle part ici on ne [puisse] échapper à l'Amérique : la méthode de forage, les lampes, jusqu'au produit distillé, tout appartient à l'Amérique », il se console à la pensée qu'il pourra visiter le lendemain Sourakani. «On dit qu'il existe là, Dieu soit loué, un temple de feu ${ }^{17}$ » (p. 211).

Le caractère sacré de ce feu apparait encore plus clairement dans le passage suivant, qui vante les mérites d'une religion du feu. «Nous voici donc à l'endroit d'où le christianisme reçut son idée poétique du "feu éternel".» Le narrateur ironise une nouvelle fois sur les spéculations savantes: "Les Anciens étaient bien bornés en matière de science. Ils apprirent seulement à connaître cette eau bourbeuse, ils y mirent le feu et elle brûla, brûla éternellement. Ils rapportèrent ce feu à Mitra, au soleil, lequel brûlait aussi éternellement et était l'image de Dieu. Et cette eau devint sacrée, ils l'adorèrent, ils s'y rendirent en pèlerinage. Et quelqu'un ayant même élevé un temple au-dessus de cette source de feu, leur reconnaissance en fut très grande. » Suit une digression sur la doctrine de Zarathoustra. Le narrateur prend visiblement plaisir à placer le christianisme dans une perspective historique qui lui enlève sa valeur de religion révélée. « Nous sommes redevables, paraît-il, de bien des notions religieuses au peuple iranien [...] Bref, Zarathoustra enseigna beaucoup de bonnes choses au christianisme » (p. 212-214).

17 Alors que les autres religions sont traitées avec la même distance ironique, les "adorateurs du feu "sont présentés sous un jour nettement favorable. Après avoir décrit les avatars du zoroastrisme, le narrateur signale que «seuls quelques fidèles émigrèrent aux Indes, emportant la doctrine intacte » (p. 215). Il s'agit des Parsis de l'Inde et des Guèbres de la Perse. «Une poignée de ces derniers habitèrent jusqu'à ces tout derniers temps, près de ce temple de feu, à Bakou [...] Pour ces justes, Mitra était resté le même qu'autrefois, le Dieu suprême, éternel comme le soleil et comme le feu éternel. Jamais lieu plus sacré ne s'offrit à l'homme [...] ici était le feu vivant, une sorte de soleil dans la terre, Dieu» (p. 215). Le texte rapporte ensuite que « ces parvenus de 
Mahométans » les ont persécutés, mais qu'ils ont continué à aller en pèlerinage à Bakou. Arrivés sur place, ils avaient le droit d'accéder au temple du feu et d'y occuper chacun une petite cellule où « une petite flamme de naphte brûlait perpétuellement. Et là ces Guèbres et ces Parsis se prosternaient, face contre terre, isolés du monde " (p. 216). Suit alors une rupture brutale dans le texte: «Beuglement : c'est l'Amérique qui débarque.» C'est l'époque où commence l'extraction du pétrole. "Tous les petits soleils des cellules étaient éteints, tous les courants de gaz avaient été branchés sur l'usine.» La conclusion du chapitre est éloquente: «Les parvenus de l'Orient les avaient persécutés, mais les parvenus de l'Occident les vainquirent [...] Leur sanctuaire de Bakou n'est plus qu'une légende. Mais le feu vivant leur restera sacré jusqu'au jour où le dernier de ces croyants aura disparu. Car ce sont les adorateurs du feu » (p. 216). La « dévotion » qui transparaît au travers de ces lignes invite à penser que le narrateur est lui-même un adepte de cette religion du feu, à laquelle il attribue évidemment un sens symbolique. Ce feu sacré et éternel dont la présence est signalée dès le début du récit, et qui représente le Caucase, c'est celui dont Prométhée s'est emparé, et le naphte, cette eau en feu devenue sacrée, représente elle aussi la source à laquelle puise l'inspiration de l'écrivain.

\section{Un intérêt marqué pour les chevaux}

Si le symbolisme du feu permet d'identifier un principe unifiant dans notre texte, un autre motif, celui du cheval, donne aussi au récit une cohérence inattendue. Les observations que nous accumulons ainsi montrent qu'une logique symbolique a présidé au travail de rédaction de ce récit de voyage d'un type bien particulier.

19 C'est dans les chapitres qui se rapportent à la traversée du Caucase que les chevaux sont le plus fortement présents. Il est vrai que cela n'a rien de surprenant, puisque le reste du voyage s'effectue en train ${ }^{18}$. Le chapitre II renferme toutefois déjà une allusion inattendue, qui place en quelque sorte l'ensemble de la Russie et de son peuple sous le signe du cheval. Alors qu'il est assis dans un restaurant, le narrateur observe les personnes qui l'entourent, et il remarque surtout une jeune fille en qui il voit « un monde » : elle a le "regard sombre et mystérieux, lourd et profond», et il se dégage d'elle une forte impression de tendresse. Le texte précise curieusement qu' « elle a ce bon et fervent regard d'une jeune jument allaitant son poulain ». L'auteur ajoute qu'elle a les pommettes saillantes, qu'il a lu quelque part que c'est un trait particulier de la physionomie des Slaves et que " ces pommettes accusées donnent à leur visage un air chevalin» (p. 27-28). Quand on tient compte de l'admiration dont les Slaves font l'objet dans l'ensemble du récit, on peut considérer que ces détails n'ont rien de gratuit.

C'est à partir de Vladicaucase, au chapitre $\mathrm{v}$, que le périple se poursuit en voiture à chevaux. Le voyage en chemin de fer ne reprendra qu'à Tiflis, au chapitre XII. Ce n'est sans doute pas un hasard si, le premier jour, le départ est retardé à cause d'une épidémie chevaline! Tout contribue à faire jouer au cheval un rôle central dans toute cette partie du texte. Lors d'un arrêt en pleine montagne, le narrateur et sa compagne remarquent que l'un des chevaux n'a rien à manger et ils prennent alors soin de donner à la bête « lésée » un peu de la ration de maïs réservée aux autres. À une autre occasion, le touriste-narrateur fait en sorte d'étendre la longe de deux chevaux pour qu'ils puissent brouter un peu d'herbe verte et il les caresse de la main. Il observe à ce moment-là que les chevaux le suivent des yeux, et il en conclut qu' « ils se sentent seuls 
et aiment la compagnie» (p. 99). Lors d'une promenade à cheval improvisée, il fait en sorte de modérer le trot de la bête "pour qu'elle ne fût pas en sueur" (p.113). L'attention affectueuse qu'il porte aux chevaux ressort encore plus clairement dans une remarque sur l'allure à laquelle roulent les voitures. Il précise que «le Finlandais nourrit bien sa bête, mais la mène durement ", et il indique aussi que, pendant un séjour en Finlande, il s'est tellement apitoyé sur le sort des chevaux qu'on l'a traité de "pleure-cheval».

Pour se rendre à Tchorny Gorod, faubourg de Bakou, il est encore obligé d'avoir recours aux services d'un voiturier qui mènent ses chevaux à un train d'enfer, et il cherche alors à lui faire comprendre "que les chevaux étaient nos semblables; selon les analyses les plus récentes ils auraient même une âme, et c'était donc presque des hommes » (p. 198). Son attachement à la race chevaline est tel qu'il est même admiratif devant le stoïcisme d'un cheval à l'air abruti, dont les plaies répandent une odeur infecte, et qui persiste à griller au soleil en clignant des yeux. Le stoïcisme de l'animal, qu'il désigne comme étant un "cheval supérieur», est comparable au "calme admirable » de la population musulmane du quartier asiatique de Tiflis (p. 183-184).

Pour conclure sur ce point, on notera une autre occurrence des images de chevaux, très évocatrice, qui place en quelque sorte la littérature toute entière sous le signe du cheval, « image de la beauté accomplie », symbole de majesté ${ }^{19}$. Il s'agit de l'analyse que le narrateur fait de la littérature russe, au chapitre Ix. On y apprend qu'il est prêt à échanger une douzaine de mauvais auteurs contre "le seul cheval de Marengo" (p. 137), le coursier d'un de ces héros hamsuniens à la forte personnalité qui savaient se faire obéir ${ }^{20}$.

Le fait que le texte renferme d'aussi nombreuses allusions à des chevaux est déjà remarquable en soi. Cette présence se fait littéralement obsédante au chapitre VII. C'est l'analyse de ce passage qui va montrer que le motif du cheval a quelque chose de très révélateur au point de vue symbolique. On sait qu'au regard de la psychologie des profondeurs, le cheval symbolise les composantes animales de l'homme et qu'il est à ce titre la «monture privilégiée de la quête spirituelle ${ }^{21} »$.

\section{Le « sacrifice » du cheval et son importance symbolique}

Ce n'est sans doute pas un hasard si les « faits ${ }^{22}$ » rapportés au chapitre VII sont censés se passer très peu de temps avant que les touristes atteignent le point le plus élevé de leur voyage, qui se situe à près de trois mille mètres (p. 116). Dans l'économie du récit, ce passage doit en effet être considéré comme un " point culminant ». On notera que la fascination que le décor exerce sur l'esprit du narrateur est telle qu'il voudrait y établir sa demeure. «J'aurais voulu demeurer ici, parmi la lune et les étoiles; qui sait, des êtres nés des nuages viendraient me voir ». L'importance capitale que ces lieux revêtent pour son psychisme apparaît à l'évidence quand il signale qu'il est prêt à baptiser cet endroit «la Source » et il ajoute: "à cause qu'il s'élève des profondeurs; voyons, une source, cela ne veut pas dire qu'on manque d'eau...» (p.101). L'émotion qui accompagne l'évocation de cet épisode invite à penser que cette «source » a un rapport direct avec un point nodal de la psyché de l'auteur. 
La première partie du chapitre décrit une scène étrange et barbare : le dépeçage d'un cheval crevé et l'espèce de repas rituel auquel il donne lieu ${ }^{23}$. Le cheval est mort parce que son propriétaire l'a fait trop boire au cours d'une ascension difficile en montagne. Du début à la fin du récit, le narrateur n'est pas en très bons termes avec son cocher. Il l'a sélectionné par qu'il fait partie de la secte des Molokans, « qui proscrit l'alcool sous n'importe quelle forme » et astreint ses membres à boire du lait ${ }^{24}$ (p. 68), mais il est constamment en conflit avec lui sur des questions d'horaires ou parce qu'il n'apprécie pas sa façon de conduire son équipage. Alors que les populations locales - musulmanes - savent utiliser l'eau de façon adéquate pour le plus grand bien de leurs chevaux, en les faisant baigner dans un fleuve (p. 80) ou dans la mer Noire (p. 242), le Molokan chrétien - fait mourir son cheval en lui administrant de l'eau froide sans discernement! Contrairement au narrateur, le cocher n'a pas un rapport harmonieux avec les chevaux.

Toute la première partie du chapitre place l'épisode de l'équarrissage sous le signe du paganisme, qui est explicitement opposé au christianisme et présenté sous un jour tout à fait favorable. En bon chrétien, le Molokan refuse de s'associer à ce rituel, mais il se laisse tout de même fléchir quand on lui offre de l'argent. C'est l'occasion pour l'auteur de rappeler le banquet sacrificiel de Lade, passage célèbre de la saga de Hakon le pupille d'Athelstan (Haakon Adalsteinsfostre) ou Hakon le Bon, qui figure dans l'Histoire des rois de Norvège (Heimskringla) de Snorri Sturluson. Rappelant que Hakon a fini par accepter un compromis en ne mangeant «que quelques bouchées de foie de cheval» (p. 93), le narrateur estime que Corneille Grégorévitch, son cocher, a eu à cet égard bien des devanciers et qu'il aura certainement aussi beaucoup de successeurs. Le narrateur a participé quant à lui à la «bacchanale » et les morceaux de viande qu'on lui a donnés l'ont même provisoirement guéri de sa fièvre. Il s'attarde avec insistance sur le "christianisme caucasien ». "Ici comme en bien d'autres pays, une des premières choses que l'on enseignât aux chrétiens était de s'abstenir de manger de la viande de cheval. » Il souligne que des ruines d'églises rappellent l'époque de la reine Tamara ${ }^{25}$, mais il rappelle aussi qu'« à Bakou il existait encore, il y a une génération à peine, des adorateurs du feu, et dans la Caucasie méridionale, vers l'Arménie, vivent toujours, paraît-il, des adorateurs du Diable»(p.90). Nous avons déjà vu que le culte du feu occupe une place privilégiée dans son esprit. Le «sacrifice» du cheval, qui est décrit avec force détails répugnants, est à mettre dans la même rubrique. Le narrateur tient à signaler qu'il se sent parfaitement en phase avec la mentalité primitive des populations païennes. "Je ressens un bien-être exquis, parmi ces hommes et ces bêtes, dans la nuit étoilée. Il me semble avoir trouvé un bon coin familier dans cette contrée lointaine " (p. 94). Le rituel en question le fascine, comme le Molokan, d'ailleurs, qui, malgré ses réticences de principe, "surveille le travail d'équarrissage » tandis que "jusque dans ses yeux à lui une lueur semble ressusciter » (p. 91).

27 L'apologie du paganisme n'épuise pas le sens de ce long passage. L'atmosphère presque mystique qui entoure la description invite à lui attribuer un fort contenu symbolique. Il $\mathrm{y}$ a tout lieu de rapprocher cette scène du motif du « sacrifice du cheval », bien connu des spécialistes de la psychologie des profondeurs. Dans plusieurs civilisations de cultivateurs, le sacrifice du cheval est en rapport avec l'idée de renouveau, de nouvelle germination ${ }^{26}$. Cet aspect de la question mérite déjà d'être signalé, car c'est bien un renouvellement psychologique que provoque le voyage au Caucase, mais l'une des toutes dernières images du livre doit aussi être mentionnée dans ce contexte. « Assis à 
la fenêtre ouverte, je regarde des hommes nus qui, à califourchon sur des chevaux, les mènent à l'eau dans la mer Noire. Leurs corps sont bronzés sur le fond bleu de la mer » (p. 242). En réalité, cette image se rattache au motif du sacrifice du cheval, dont une variante se fait par immersion dans les eaux d'un fleuve. Le Dictionnaire des symboles souligne l'affinité du cheval et des eaux courantes et rappelle qu'en tant que « divinité des eaux ", cet animal passe pour avoir «le don de faire jaillir des sources du choc de son sabot [...] Pégase lui-même inaugure cette tradition en créant la source Hippocrène - Source du cheval - non loin du bois sacré des Muses ; les Muses s'y réunissaient pour chanter et danser, son eau passait pour favoriser l'inspiration poétique " (op. cit., p. 230). On note donc que le cheval éveille ici l'Imaginaire, comme il éveille la nature, au moment du renouveau. La conjonction du cheval et de la source figure expressément dans le chapitre qui fournit la matière de ce développement. L'ultime image des hommes qui mènent des chevaux à la mer confirme l'idée qui nous semble sous-tendre l'ensemble de l'ouvrage et que nous défendons dans cet article: l'inconscient de l'auteur y a révélé où étaient les sources de son inspiration.

Une fois qu'on a noté ce détail, il est intéressant de pousser l'analyse un peu plus loin. On s'aperçoit alors que ce passage permet sans doute de passer de la perspective du narrateur - le récit est écrit à la première personne - à celle de l'auteur, et qu'il donne sans doute accès à l'inconscient personnel de Knut Hamsun. Comme le signale l' Encyclopédie des symboles, « la psychologie voit dans le cheval, au niveau le plus profond, un symbole chtonien à connotation maternelle, qui figure les couches les plus profondes de l'inconscient dans leur double aspect créateur et destructeur ${ }^{27}$. " Dans Métamorphose de l'âme et ses symboles, dans un chapitre intitulé «La lutte pour se délivrer de la mère " (p. 455-507), C.G. Jung rappelle par ailleurs que «l'image de la mère est un symbole de libido comme le cheval» (p. 456) et accorde une large place au cheval en tant que symbole. Il décrit surtout comment un sacrifice est indispensable pour que l'âme puisse parvenir à l'indépendance personnelle. «Ce sacrifice signifie vraisemblablement abandon de l'attachement à la mère, c'est-à-dire renoncement à tous les liens et toutes les limitations que l'âme a emportés depuis l'enfance jusqu'à l'âge adulte [...] La vie appelle l'homme au dehors, à l'indépendance et quiconque, par commodité ou crainte infantile, n'obéit pas à cet appel est menacé de névrose. Une fois que celle-ci a éclaté, elle deviendra progressivement une raison plus que suffisante pour fuir le combat de la vie et rester à jamais embourbé dans la prison morale de l'atmosphère infantile » (p. 504-505).

29 C'est précisément à ces couches de la psyché de l'auteur que semble donner accès la deuxième partie du chapitre VII de Au pays des contes. Tout se passe comme si ce long passage venait confirmer - comme un sorte de commentaire plus personnel l'impression générale laissée par la lecture symbolique de la première ${ }^{28}$.

\section{En pénétrant dans la montagne}

30 Nous sommes arrivés au moment où le narrateur décide de faire une promenade à cheval. On notera qu'il s'en va alors «dans la montagne ${ }^{29}$ » (p. 99). La formulation norvégienne est très claire, il part à la découverte d'endroits reculés dans le massif montagneux, mais pris littéralement, elle pourrait signifier qu'il "pénètre" directement dans la matière rocheuse elle-même. C'est d'ailleurs ce qu'il fait littéralement lorsqu'il rend visite à un berger, puisque l'habitation de celui-ci a été 
creusée dans le roc, de même que l'étable où il se rend pour finir. On sait que dans le monde des contes et de la mythologie nordiques, pénétrer dans la montagne, c'est entrer dans un monde fantastique et inquiétant, c'est s'exposer à quelque sortilège. C'est bien le risque que prend le narrateur. Il n'est pas très rassuré et, par deux ou trois fois, il est même saisi par la peur et croit que sa dernière heure est arrivée. Il est toutefois prêt à en découdre et souligne curieusement qu'il est "d'une force herculéenne ». " Il ne m'aurait pas déplu que quelqu'un de chez nous m'eût vu pendant cette terrible lutte avec un sauvage» (p.101). Le texte semble signaler ici qu'il est important pour lui d'en imposer à son entourage familier. La peur qu'il ressent indique certainement aussi qu'il aborde une phase périlleuse de son périple «initiatique». Comme dans le mythe du héros, il pénètre dans une cavité sombre, mais un feu ou une lumière providentiels viennent l'éclairer. Quels sont les dangers qu'il affronte?

31 La grande découverte qu'il fait dans la première de ces cavernes, celle où habite le berger tatar, c'est « quelque chose qui grouille dans un coin », qui n'est ni plus ni moins que la mère du propriétaire des lieux, une vieille femme "ratatinée » et aveugle. On notera le commentaire du narrateur : «Et je me rappelais avoir lu que les Caucasiens ne se soucient pas le moins du monde de ce que désirent leurs femmes, mais se rangent docilement à l'avis de leur mère. » On s'aperçoit en effet que le berger suit docilement les injonctions de sa mère. Le visiteur se montre d'ailleurs très avenant à son égard, lui aussi, mais il explique sa cécité par une comparaison avec les chevaux et les vaches : lorsqu'on les attache trop près du mur, «ils en deviennent myopes. Et je me dis : la femme caucasienne connaît un sort semblable; on l'attache trop près du mur. Et elle devient aveugle» (p. 105). Le narrateur est resté en bons termes avec la vieille mère, avec qui il a partagé une entrecôte de mouton grillée sur " un feu admirable », mais cela ne résout pourtant pas le problème de ses rapports avec le monde féminin.

Il s'intéresse en effet surtout à ce qu'il appelle le « harem » du berger, qui semble être composé de deux femmes. Tout se passe comme si la normalisation du rapport à la mère avait été une étape indispensable à la découverte de la femme comme partenaire. Il est intéressant de noter que le passage suivant est l'un de ceux où le narrateur laisse le plus librement cours à sa fantaisie débridée. Fait symptomatique, il imagine que c'est par le biais d'un poème qu'il va pouvoir séduire celle qu'il croit être la favorite de son hôte. Ces quelques vers traitent curieusement du statut de la femme et il espère qu'ils vont « donner le branle à tout un petit mouvement féministe en Caucasie » (p. 106-107) et même devenir la chanson nationale du pays. Ils sont supposés inspirer à la femme du berger « de l'estime pour un homme qui saurait faire de si drôles de jambages sur du papier ». Le troisième vers se rapporte aux enfants, qui sont désignés comme «le mal béni » (forbandelsens velsignelse, c'est-à-dire littéralement: la bénédiction de la malédiction) et semble anticiper sur la visite dans la deuxième grotte. Le narrateur estime que ce détail posera une énigme à la jeune femme.

Une fois ce projet échafaudé, il a peur que le berger ne se rende compte de ses intentions et il craint de le suivre lorsque celui-ci l'invite à entrer avec lui dans l'autre caverne. Il a alors le sentiment que la jeune femme est de connivence avec son mari et, pour montrer ce dont il est capable, il finit par pénétrer dans ce qui va se révéler être une simple étable. Arrivé dans l'obscurité de cette grotte, il est presque pris de panique et s'apprête à se défendre, jusqu'au moment où il comprend que celui qu'il prend pour un assassin veut simplement lui vendre deux oursons. Maintenant qu'il a pu constaté que le pauvre Tatar n'a "que de paisibles intentions de commerce", il retrouve sa 
supériorité. Il refuse le prix qu'on lui propose et, estimant avoir ainsi obtenu de « riches résultats scientifiques", qu'il lui faudra "au moins quatre ans ${ }^{30}$ " pour exploiter, il estime que sa mission est terminée et il regagne son cheval, le caresse et précise curieusement : «J'étais son maître ». Cette maîtrise du cheval s'affirme effectivement sur le chemin du retour puisqu'il prend soin de « modérer le trot de la bête pour qu'elle ne fût pas en sueur » (p. 113).

L'ensemble des détails résumés ci-dessus semblent se prêter à la brève analyse suivante. Nous croyons pouvoir lire ce passage comme une introspection dans les couches profondes de la psyché de l'auteur et y voir le reflet de la fixation maternelle qui semble l'avoir profondément marqué. Nous nous appuyons ici sur les résultats des recherches de Lars Frode Larsen (Den unge Hamsun 1859-1888, 1998), pour qui le traumatisme majeur subi par le jeune Hamsun n'a pas été l'oncle Hans Olsen - souvent décrit comme particulièrement tyrannique - mais bien les expériences qu'il a faites dans son propre foyer, dans un grand dénuement matériel, auprès d'une mère en proie à une névrose profonde ${ }^{31}$. Pour expliquer le fait que Hamsun lui-même n'ait pratiquement jamais fait allusion à ces faits, on sait que Lars Frode Larsen s'appuie sur l'idée qu'il a idéalisé ses parents et l'éducation qu'ils lui ont donnée et que ce fort blocage l'a amené à projeter sur son oncle le ressentiment profond qu'il a conçu quant au traitement qu'on lui a fait subir. On peut considérer que la vieille mère aveugle pourrait représenter celle de Hamsun. Nous avons vu que le narrateur se montre avenant à son égard, mais il cherche avant tout à gagner les bonnes grâces de la jeune épouse du berger, comme s'il s'agissait de franchir une nouvelle étape dans la découverte de l'éternel féminin. Nous avons également remarqué que c'est bien cette perspective qui déclenche l'inspiration poétique. La deuxième caverne permet une découverte tout aussi importante. Peut-on voir dans les deux oursons l'image du jeune Knut et de sa sœur Sophie, placés l'un et l'autre auprès de l'oncle Hans Olsen à la suite d'une sorte de "transaction commerciale» qui a profité avant tout aux parents? L'évidente satisfaction du narrateur à la suite de cet épisode semble signaler qu'un problème est résolu, un blocage a été identifié. Cette "plongée » dans les profondeurs de la psyché de l'auteur ne débouche pas sur une prise de conscience nette, mais elle a au moins permis de formuler un problème de façon inconsciente.

\section{Le Nordland : un « Caucase » intérieur}

L'avant-dernier paragraphe du texte renferme deux images: celle des hommes qui mènent leurs chevaux à l'eau dans la mer Noire, déjà citée, et celle des ruines du château de la reine Tamara, qui rappelle le caractère féerique du décor dans lequel le récit s'est déroulé. Ces images se rattachent aux deux dimensions du texte, la réalité vécue et le rêve. En évoquant le souvenir d'une reine de légende et de ses châteaux fabuleux $^{32}$, l'auteur rappelle une dernière fois que le Caucase est effectivement à ses yeux un pays merveilleux, tandis que la métaphore des "chevaux aquatiques " fait allusion - nous venons de le voir - à l'inspiration poétique. Ce pourrait être une façon de signaler que le " pays des contes » est à la fois le Caucase " géographique » et le pays de l'enfance de l'auteur, qui a inspiré pratiquement toute son œuvre. Plusieurs passages établissent une nette corrélation entre le décor qui provoque l'émotion du narrateur et le Nordland où Hamsun a grandi. 
C'est au chapitre VI, au moment où commence le voyage en voiture, que le narrateur le signale pour la première fois expressément. «Ce coin nous frappe par son caractère absolument norvégien... » (p. 74). Il ajoute que « notre cher bouleau du Nord ne connaît pas la fatigue ; il grimpe jusqu'au sommet, alors que tous les autres arbres, pris de froid, s'arrêtent » (p. 76). Nous avons mentionné par ailleurs l'impression magique que le glacier de Kasbek lui a faite. Au moment où cette "vision » vient de disparaître, il enchaîne, " abasourdi », sur un souvenir du temps de son enfance, en Nordland, où, au cours d'une «nuit étrange » où il se déplaçait en barque, une tête d'animal, sans doute un phoque, émergea de l'eau, « on aurait dit je ne sais quel être d'un autre monde. Les yeux ouverts, il me contemplait; son regard était pareil à un regard humain » (p. 79). Le même phénomène se reproduit dans le chapitre suivant, à un degré plus fort encore, après la «bacchanale » déjà citée. La majesté des montagnes impressionne le narrateur. "La tête renversée, je regarde leurs sommets qui se profilent sur le ciel. Je regarde aussi les étoiles. » Le souvenir du Nordland s'impose alors à lui avec force, même s'il précise que la beauté du spectacle l'« empêche de ressentir de la nostalgie ». Fixant les yeux sur le ciel étoilé qui l'ensorcelle littéralement, il se laisse entraîner dans une méditation métaphysique. "Comme je suis de ceux-là qui, au contraire de bien d'autres, ne sont pas encore fixés sur la question [de] Dieu, je reste un moment à penser à Dieu et à sa création. " Il ne livre pas le résultat de ses cogitations, mais on a le sentiment qu'il se laisse aller à quelque communion mystique avec la nature qui n'a rien à voir avec le Dieu de la Bible. "Comme tous ceux qui ont vécu solitaires, je m'entretiens avec moi-même, à l'excès, selon mon habitude - je m'accroupis, frissonnant de joie et parlant à haute voix. Je voudrais dormir ici. Et je m'étends sur le dos, je frétille, me réjouissant dans mon corps que tout soit si bien » (p. 98-99).

Avant d'en venir aux réminiscences rapportées au chapitre IX, qui sont les plus éloquentes dans la perspective où nous nous plaçons ici, il est utile de souligner que, dès le début du récit, le lecteur a été préparé à l'idée que la solitude induit chez le narrateur une «surexcitation intérieure » (p. 28). Le chapitre II nous montre comment il part seul à la découverte des environs de son hôtel, sous le prétexte de trouver un tailleur qui pourra recoudre un de ses boutons. Arrivé dans un cabaret, le contact avec la population locale lui fait l'effet d'un « enchantement » (trylleri, le mot s'emploie pour désigner la magie), et il est tout à coup « extrêmement joyeux ». Il se remet à parcourir les rues en toute liberté. "J'ignore où je suis, je ne sais quelle direction prendre. Sensation exquise, je me suis égaré ; personne ne saurait en comprendre le charme, s'il ne l'a éprouvé. J'use délibérément de mon légitime droit de me perdre. » Après être entré dans un restaurant, il se sent toujours « heureux et libre ». " Il me semble avoir trouvé une cachette, où je peux, à loisir, passer tout mon temps [...] Bref, ici c'est à moi qu'il appartient de prendre telle résolution qu'il me plaira et de grand cœur je remange de la viande » (p. 26-28). On peut supposer que son sentiment de liberté s'explique en partie par le fait qu'il se promène alors sans sa compagne de voyage et qu'il peut ainsi agir à sa guise. Les réflexions qu'il fait à cette occasion ont toutefois une portée bien plus générale. «À cette table, je me trouve chez moi, c'est-à-dire loin de chez moi, donc à merveille. » L'ensemble de ce passage indique que le sentiment de solitude absolue et d'isolement produit un effet particulier sur son psychisme.

C'est exactement ce qu'il explique au chapitre Ix. « Nulle chose au monde n'égale, oh! non, la sensation d'être loin de tout, pensé-je encore.» Et il greffe aussitôt sur cette remarque des souvenirs d'enfance particulièrement révélateurs pour notre propos. « Le 
souvenir m'en revient de mon enfance, des années où je gardais mon troupeau, là-bas, au pays. Par le beau temps, allongé sur le dos dans la bruyère, j'écrivais tout en travers du ciel avec mon index et je passais de divines journées [...] Ceux qui n'ont pas vécu cette vie dès leur jeune âge ne peuvent se figurer l'exquis et singulier plaisir que l'on éprouve à se trouver dehors par la pluie, assis à l'abri, sous un rocher. Plus tard j'ai essayé de traduire ces impressions, mais j'y ai mal réussi. J'ai voulu y mettre du style, mais alors ce n'était plus ça. Quand j'étais berger, j'allais en sabots et, par les jours de pluie, j'avais naturellement les pieds mouillés. Or, la jouissance de sentir le bon bois chaud sous la plante des pieds, malgré que je fusse trempé, dépasse dix autres jouissances ultérieures. [...] Je suis dans un état d'esprit singulier, je sens que je pourrais très bien prendre racine par ici et m'isoler délicieusement du monde » (p. 132-134). Un poème publié la même année que Au pays des contes, dans le recueil Le chœur sauvage, indique que l'auteur identifie ici ce qu'il faut sans doute considérer comme une de ses toutes premières expériences de création littéraire. La scène se passe dans la forêt. Tout est calme. «Je suis allongé sur le dos et je réfléchis dans l'obscurité/ et je me sens calme et joyeux. /Je suis allongé et j'écris sur le ciel/et par-dessus l'armée des étoiles avec ma chaussure ${ }^{33}$.» On remarque que c'est là aussi la contemplation du ciel étoilé et la solitude qui l'ont poussé à écrire.

\section{Une image obsédante}

Ce n'est pas un hasard si le court texte que nous venons de citer a pour cadre la forêt. Comme Glahn, Hamsun avait le sentiment d'« appartenir aux forêts et à la solitude ». Un autre poème, intitulé « Tombe » (Gravsted, op. cit., p. 31-32), dans le recueil déjà cité, se présente comme une prière adressée à Dieu, dans laquelle l'auteur souhaite mourir subitement dans la forêt pour que son cadavre y soit dévoré par les animaux et qu'il s'endorme ainsi au sein de la nature. «Je connais bien la forêt, je suis son fils... » Cette forêt est le lieu du mystère, symbole de l'inconscient. Elle peut devenir «le lieu de l'isolement pour l'homme soucieux de spiritualité, où il est en sûreté et à l'abri de l'agitation du monde. » Elle est aussi « l'équivalent du désert où le méditatif se retire à l'écart des comédies de la vie, dans un retour vers les forces primitives où peuvent le mieux se révéler l'essence et l'unité de son âme, et où l'on se retrouve sans appel face à soi-même et au divin ${ }^{34}$. » En réalité, chez Hamsun, la forêt fait souvent partie d'une image obsédante qu'on retrouve tout au long de sa production littéraire et qui figure comme un signal - au début de Au pays des contes: l'image d'un homme qui suit un sentier envahi par les herbes. «La campagne s'ouvre toute grande devant nous. À gauche, une forêt, où un sentier serpente à travers bois, qu'un homme suit. " L'auteur signale avec insistance l'importance de cette scène pour son psychisme : "Cette image a quelque chose de si familier, et depuis si longtemps j'habite l'étranger, que j'ai plaisir à la contempler. Le sentier est à demi couvert d'herbe (igjengrodd) et l'homme qui le suit porte le sac au dos. Où s'en va-t-il si matin? me dis-je; sa besogne l'appelle probablement de l'autre côté du bois » (p. 16). Avec sa phrase interrogative, ce passage fait fortement penser aux premières lignes des Fruits de la terre (Markens grøde) : « Le long, long sentier par les marécages et les forêts, qui l'a frayé ? L'homme, l'être humain. Avant lui, pas de sentier [...] L'homme arrive, montant vers le nord. Il porte un sac, son premier sac [...] Peut-être, fuyant le châtiment, se cache-t-il ici, ou peut-être est-ce un philosophe qui aspire à la paix... » On sait par ailleurs que le tout dernier livre sorti de la plume de Hamsun est composé en grande partie de réminiscences tirées de l'enfance 
de l'enfance de l'auteur, et qu'il porte précisément le titre de Sur les sentiers où l'herbe repousse (På gjengrodde stier).

Mais c'est sans doute le chapitre introductif de Sous l'étoile d'automne qui montre le plus clairement que l'image en question peut être considérée comme un élément fondamental du «paysage intérieur » de l'auteur. « Quand je pénètre dans la forêt, par le sentier où l'herbe repousse, mon cœur tremble d'une joie non terrestre ${ }^{35}$." Ce passage qui baigne dans une atmosphère quasi mystique est d'autant plus important pour notre propos que le narrateur met cette image en rapport direct avec le voyage au Caucase. «Je me rappelle un endroit sur la côte orientale de la mer Caspienne où je fus une fois. Là, c'était comme ici, la mer était calme et lourde, gris fer, comme maintenant. Je pénétrai dans la forêt, je fus ému jusqu'aux larmes, j'étais ravi, je disais tout le temps : Dieu du ciel! Dire que je devais revenir ici ! Comme si j’y étais déjà allé ?» Le texte enchaîne alors sur l'idée panthéiste d'une communion mystique avec la nature et d'un éternel retour : le narrateur a peut-être été jadis « une fleur dans cette forêt, peutêtre étais-je un coléoptère qui logeait dans un acacia. " On sait par ailleurs que Sous l'étoile d'automne expose à la perfection le mouvement de diastole et de systole qui est si caractéristique de Hamsun et qui correspond parfaitement à l'expérience centrale que l'auteur a faite au Caucase: "Me voici loin du vacarme et de la presse de la ville, des journaux et des gens, j'ai fuit tout cela parce que, de nouveau, la campagne et la solitude dont je suis originaire m'appelaient. Je pense, plein d'espoir : Tu verras, tout va bien aller. Hélas! Je me suis déjà enfui de la sorte et je suis retourné à la ville. Et me suis de nouveau enfui. »

\section{Le pays des contes et l'eau de la Koura}

Dans un essai publié en 1910, « Le théologien au pays des contes ${ }^{36}$ ", Hamsun s'en prend avec véhémence aux pasteurs qui refusent d'exercer leur sacerdoce dans le Nordland, et il dresse un tableau très flatteur de la région où il a passé son enfance. Il souligne que ses habitants "ont une tradition riche en poésie, [...] des contes et des légendes", et que le théologien qui accepte de vivre sous ces latitudes a d'immenses avantages: les nuits étoilées, les aurores boréales, une flore "oubliée et négligée ", une fleur dans la montagne, un buisson dans la vallée, le calme, etc. Ce merveilleux spectacle «le détourne de penser grossièrement à son avantage, et l'oriente vers une vie contemplative, une vie religieuse ». Il désigne expressément le Nordland comme étant le « pays des contes » et il signale d'ailleurs dès les premières lignes de son article qu'il doit cette expression à Bernt Lie, écrivain norvégien qui a puisé un bon nombre de ses motifs littéraires dans la Norvège du nord. C'est vraisemblablement à lui que Hamsun a emprunté le titre de son livre sur son voyage au Caucase. Lie avait en effet publié en 1892 un ouvrage consacré au Nordland et intitulé Au pays des contes. On comprend mieux alors l'inquiétude que traduit Enfants de leur temps et La ville de Segelfoss: en menaçant le caractère naturel du Nordland, l'industrialisation mettait aussi en péril la source d'inspiration de l'auteur. C'est n'est pas hasard si, en décrivant la disparition du monde auquel il était tant attaché, il estime que «le conte est fini ${ }^{37}$ ». "Nous avons un roi, M. Holmengrå, [...] il a une princesse, [...] nous avons un château abandonné ${ }^{38}$. $\mathrm{Ce}$ monde, c'est l'univers de son enfance, son cadre de référence initial, le paysage intérieur qui fournit la clé de son œuvre. Le voyage de l'automne 1899 a été pour Hamsun l'occasion d'une telle «illumination » qu'une fois rentré en Scandinavie, après 
un bref séjour à Copenhague, puis à Kristiania, il fut incapable de résister à la nostalgie du Nordland et se rendit brusquement à Hamarøy, laissant sa femme seule pour quelque temps ${ }^{39}$.

Dans les deux dernières phrases de son livre, Hamsun rappelle l'eau de la Koura, qui donne à celui qui en boit une nostalgie du Caucase qui ne l'abandonnera plus. Il ne pouvait pas trouver une meilleure métaphore pour résumer son propos. Nous souhaitons avoir montré qu'elle désigne l'inspiration, le « fleuve » intérieur qui avait sa source dans l'enfance de l'auteur et la nature intacte et primitive du Nordland. Si le voyage au Caucase a permis à Hamsun de découvrir la Russie, il a été avant tout pour lui la route du Voyage intérieur, la découverte d'un Orient spirituel ${ }^{40}$ dont l'ensemble de son œuvre atteste l'importance.

\section{NOTES}

1. «Her står formlen for hans forfatterskab, den grundlæggende konflikt, en gang for alle formuleret og defineret [...], vor tids og vor klodes store drama : et fattigt liv med mening over for et rigt liv uden. » Thorkild Hansen, Processen mod Hamsun (1 ${ }^{\text {re }}$ édition, 1979), ici : Gyldendals Paperbacks, 1996, p. 44-45.

2. "Synspunktet er slett ikkje utan fundament.» Atle Kittang, Luft, vind, ingenting, Hamsun desillusjonsromanar fra Sult til Ringen sluttet, 1984, p. 134.

3. Atle Kittang lui a réservé un assez long développement dans Luft, vind, ingenting, p. 125-140. À l'automne 1999, la Société Hamsun a publié une communication de Henning Howlid Wærp: "Knut Hamsun som reiseskildrer: I Æventyrland», in: Hamsun i Tromsø II, Rapport fra den 2. internasjonale Hamsun-konferanse 1999, p. 239-261. L'année suivante, le même éditeur publiait un court texte de Bjørn Rudborg et Ole Petter Førland : «I Æventyrland. Oplevet og drømt i Kaukasien -100 år etter ", destiné à accompagner une série de diapositives sur le sujet, in : Hamsun 2000, 8 foredrag fra Hamsun-dagene på Hamarøy, p. 43-55. En 2000 toujours, les deux mêmes auteurs ont publié le texte de Hamsun, I Æventyrland. Oplevet og drømt $i$ Kaukasien, avec une postface détaillée (p. 143-201) et illustrée, à la suite d'un voyage sur les traces de l'auteur. C'est cette édition en norvégien que nous avons utilisée pour le présent travail.

4. Voir note 40. Le roman Victoria est très révélateur quant aux conditions dans lesquelles est née la vocation d'écrivain de Hamsun.

5. Les citations en français sont tirées de la traduction publiée aux éditions Bernard Grasset dans la collection «Les Cahiers Rouges ", sans nom de traducteur. La première édition date de 1936, mais celle que nous avons utilisée a été imprimée en septembre 2000.

6. Comme lorsqu'elle souhaiterait faire un brin de toilette après un voyage harassant, avant de passer à table : « Mais, connaissant mon devoir, je donne un coup sur la table : d'abord à manger, les atours et la vanité ensuite. Et je fais triompher mes volontés. » (p. 60)

7. On apprend d'ailleurs dans un autre passage (p. 94) qu'il ne s'en est débarrassé que sur la route du retour, en le jetant par la fenêtre du train. Quelque chose de capital se sera alors produit. Un blocage aura été levé, ou pour le moins identifié, comme nous aurons l'occasion de le voir plus loin.

8. Dans un autre passage du texte, il signale qu'il a assisté dans sa jeunesse à des conférences données par Georg Brandes. "Je me sens redevenir, un moment, tout jeune, je regarde émerveillé 
devant moi et j'entends battre mon cœur. J'éprouve la même sensation qu'au jour où, pour la première fois, j'entendis Georges Brandès faire une conférence." Il conclut sur une remarque désobligeante qui en dit long sur ce qu'il pensait de celui qui a été l'un des plus importants maîtres à penser de la Scandinavie moderne : «Et il monta sur l'estrade... Au reste, j'avoue que j'aurais bien mieux discouru moi-même. C'est entendu» (p. 164-165). Comme ce souvenir lui revient au moment où il parle de Tiflis, «que tant de poètes russes ont chantée et où se sont passés tant d'événements du roman russe » (p. 164), et qu'il compare expressément l'émotion qu'il ressent alors à celle qui s'est emparé de lui au moment où il devait entendre le grand comparatiste danois, on doit certainement comprendre qu'il veut placer sa "découverte " audessus de celle de Brandes et qu'il se pose plaisamment en chef de file d'un mouvement d'une inspiration tout à fait différente.

9. Plusieurs détails semblent l'attester, et sa mère l'a confirmé dans une interview de 1911 : « Il écrivait toujours. Déjà étant enfant, il écrivait des vers sur ses camarades et il dessinait des feuilles et des fleurs. Il n'avait pas toujours un support ou un instrument avec lesquels il pouvait écrire, mais il n'était jamais à court d'idées. Il écrivait et dessinait dans la neige et gravait des inscriptions sur les arbres. Le soir, au clair de lune, il lui arrivait de rester debout à la fenêtre dans la chambre des garçons, après que les autres s'étaient couchés, alors qu'il faisait noir, et d'écrire sur la vitre, qu'il recouvrait de buée en soufflant dessus. » Cf. Lars Frode Larsen, Den unge Hamsun 1859-1888, 1998, p. 68-69.

10. Au sens figuré, pour l'instant, mais c'est presque vrai au sens propre aussi, comme le lecteur l'apprend peu après (p. 116).

11. Au moment où le train entre dans la région du Don, l'attention du narrateur est attirée par « une grosse tache sombre, à une faible distance du sol. On dirait un ballon, figé sur la prairie et qui ne monte pas. » Il apprend alors qu'il s'agit d'un grand arbre et il signale que « des lieux à la ronde on l'appelle "l'arbre" » (p.36). On est tenté d'accorder à ce curieux arbre une valeur d'archétype. Il pourrait symboliser l'axe du monde, comme l'Yggdrasil de la mythologie nordique, et souligner l'importance du sol russe pour l'univers imaginaire de l'auteur.

12. Cf. Knut Hamsun, De la vie inconsciente de l'âme et autres textes critiques, trad. Régis Boyer, 1994, p. 146.

13. C'est curieusement l'idée principale que Hamsun semble avoir voulu défendre dans sa pièce La reine Tamara, parue la même année que Au pays des contes et au demeurant fort médiocre.

14. Cf. l'article « Orient-Occident » in : Encyclopédie des symboles, collection Pochothèque, Le Livre de Poche, p. 483-484.

15. On peut considérer que la "fièvre caucasienne " dont souffre le narrateur place aussi le voyage sous le signe du feu.

16. La traduction française que nous utilisons ici coupe le chapitre en deux. Le texte auquel nous nous référons dans ce passage correspond donc aux chapitres XVI et XVII de la version française.

17. Il serait préférable de traduire par « temple $d u$ feu ».

18. Notre analyse étant centrée sur ce qui nous paraît être l'aspect principal du texte, nous ne nous attardons pas ici sur la valeur symbolique du train (cf. article «train » in : Dictionnaire des symboles), des contrôles de billets, et autres détails dont il faudrait tenir compte pour une lecture psychologique exhaustive. La surveillance policière dont le narrateur fait l'objet au cours du voyage pourrait aussi fournir un élément de réflexion intéressant pour une lecture psychanalytique de stricte obédience.

19. Cf. Dictionnaire des symboles, p. 231.

20. L'admiration de Hamsun pour les personnes qui ont le don du commandement apparait clairement dans Au pays des contes. Le tsar et Napoléon sont cités expressément. " On obéit à un homme qui sait commander. On obéissait à Napoléon avec ravissement. C'est une jouissance que d'obéir. Et le peuple russe sait encore obéir » (p. 53). 
21. Jean Chevalier, Alain Gheerbrant, Dictionnaire des symboles, Mythes, rêves, coutumes, gestes, formes, figures, couleurs, nombres, édition 1982, p. 225.

22. Nous sommes en réalité dans un univers nettement onirique qui justifie la lecture que nous proposons ici.

23. On trouve plusieurs scènes tout à fait comparables dans le roman Kongens Fald (La chute du roi), de Johannes V. Jensen, paru en 1900-1901. Il est fort probable que Hamsun l'ait lu. Le romancier danois était l'un de ses amis les plus proches à l'époque.

24. De son côté, le touriste-écrivain fait une forte consommation de cognac, qu'il boit dans des verres à bière. Il en donne même à un mouton malade !

25. Cf. notre note 13 .

26. Cf. Dictionnaire des symboles, p. 227-228.

27. Cf. Encyclopédie des symboles, La Pochothèque, Le Livre de Poche, p. 130.

28. Nous tenons à rester d'autant plus prudent que cette période de la biographie de l'auteur est assez mal connue. Les remarques que nous ferons ici ont ceci d'intéressant - nous le verrons plus loin - qu'elles semblent confirmer certaines analyses les plus récentes sur la petite enfance et l'adolescence de Knut Hamsun.

29. L'original met l'expression au pluriel : « ind i fjældene ».

30. Le plus étrange est que Hamsun a publié son récit en 1903, soit quatre ans après le voyage au Caucase.

31. L'article de Rolf Steffensen (in: Hamsun 2000, 8 foredrag fra Hamsun-dagene på Hamarøy, 2000, p. 100-121) intitulé « Knut Hamsun og den ubrukte nøkkelen » (K.H. et la clé inutilisée), qui se fonde en bonne partie sur les théories d'Alice Miller, va dans le même sens, tout en insistant davantage sur la « pédagogie noire » dont le jeune Knut aurait été victime et le rejet dont il a fait l'objet de la part de ses parents.

32. On trouve par ailleurs au chapitre $v$ une description des restes du palais d'un souverain oriental (p. 205), qui font évidemment penser à un décor digne des Mille et une Nuits.

33. « Høstnat », in : Knut Hamsun, Det vilde Kor og andre dikt, édition 1999, Gyldendal, p. 36.

34. Cf. l'article "Forêt" in: Encyclopédie des symboles, La Pochothèque, Le Livre de Poche, p. $272-274$.

35. Toutes les citations de ce paragraphe sont tirées de Sous l'étoile d'automne, trad. R. Boyer, Le Livre de Poche, p. 7-8.

36. Gunvald Hermundstad (red.), Hamsuns polemiske skrifter, 1998, p. 175-179.

37. «Men æventyret var ute. » Knut Hamsun, Segelfoss by, Gyldendal Pocket, p. 85. Nous nous permettons de renvoyer ici à notre article : « Det gamle samfunnets undergang - eller eventyrets slutt » in : Régis Boyer og Nils M. Knutsen (red.), Hamsun i Paris, 8 foredrag fra Hamsun-dagene i Paris 1994, Hamsuns-Selskapet 1995, p. 69-85.

38. «Vi har en konge, Hr. Holmengrå [...] han har en prinsesse [...] Vi har et forlatt slott. » Ibid., p. 32 .

39. Tore Hamsun, Knut Hamsun - min far, édit. 1992, p. 184.

40. Le roman Victoria, publié en 1898 , le confirme. L'enfance de l'auteur y joue le même rôle que dans notre analyse. C'est dans la réponse que fait le personnage principal, l'écrivain Johannes Møller, à un toast prononcé en son honneur, qu'on trouve le passage le plus significatif pour notre propos. Les paroles qu'il prononce sont rapportées au discours indirect. « Sa place était ici, les forêts du château était tout son univers, et derrière, baigné dans une lumière bleue, il y a avait le pays inconnu, le conte.» Op. cit., Gyldendal Pocket, p. 67. C'est nous qui soulignons. Dès les premières pages du roman, le lecteur apprend que le futur écrivain a vécu dès son enfance dans un monde de contes de fées, par lequel il a cherché à échapper à sa condition modeste, qui lui interdisait de faire pleinement partie de l'univers du château. Au cours de son bref discours, Møller rappelle aussi qu'il a gardé un souvenir inoubliable des jeux de son enfance. En réalité, c'est seulement à Victoria qu'il pense, mais il est plus prudent de sa part de parler des deux 
enfants de la famille des châtelains, puisqu'il assiste alors au repas de fiançailles de la jeune fille. «... ces moments avaient eu pour sa vie une importance que personne ne connaissait, et si comme on venait de le dire - ce qu'il écrivait répandait parfois des flammes, cela venait justement $\mathrm{du}$ fait que les réminiscences de cette époque l'enflammaient; c'était le reflet du bonheur que ses deux camarades lui avaient procuré dans son enfance. » Le commentaire de la châtelaine en dit long : «C'est bien compréhensible. Il était si seul étant enfant. »

\section{RÉSUMÉS}

Le but de cet article est de montrer que le voyage que Knut Hamsun a fait avec sa première femme en 1899 au Caucase a été surtout une sorte de voyage initiatique qui lui a révélé l'importance des paysages de son enfance pour son psychisme et pour son inspiration. Le décor de conte de fées qu'il a découvert en Russie lui a fortement rappelé le Nordland où il a grandi. Plusieurs images obsédantes sont particulièrement révélatrices: le feu, qui symbolise l'inspiration et qui hante une bonne partie du texte, le cheval, très présent dans les pages consacrées aux montagnes du Caucase, et dont le sacrifice signale une renaissance, et le sentier envahi par la végétation et que suit un homme qui se rend dans une forêt mystérieuse, motif que l'on rencontre au début de ce texte mais aussi dans un bon nombre de romans de Hamsun et jusque dans sa toute dernière œuvre, Sur les sentiers envahis par la végétation (På gjengrodde stier).

Anhand dieses Artikels wird gezeigt, dass die Reise, die Knut Hamsun 1899 mit seiner ersten Frau im Kaukasus gemacht hat, vor allem eine innere Reise, eine Art Einweihung gewesen ist, die ihn die Bedeutung der Landschaft seiner Kindheit fur seine Inspiration zumindest intuitiv hat erkennen lassen. Die märchenhafte Landschaft, die er in Russland entdeckt hat, hat ihn stark an das Nordland erinnert, wo er aufgewachsen war. Mehrere Bilder, die sich wie zwanghaft wiederholen, sind besonders aufschlussreich: das Feuer, das die dichterische Inspiration symbolisch darstellt, das Pferd, das in den Abschnitten, in denen vom Kaukasus die Rede ist, häufig vorkommt, und dessen Opferung eine psychische Wiedergeburt signalisiert, und ein Pfad, das überwachsen ist und auf dem ein Mann wandert, um in einen geheimnisvollen Wald zu gelangen, ein Motiv, das am Anfang dieses Textes sowie in manchen von Hamsuns Romanen und sogar in seinem allerletzten Buch, Aufüberwachsenen Pfaden vorkommt.

\section{AUTEUR}

\section{MARC AUCHET}

Université de Paris IV-Sorbonne 